\title{
Towards a psychophysical evaluation of a surgical simulator for bone-burring
}

\author{
Gavin Brelstaff*, Marco Agus, Andrea Giachetti, Enrico Gobbetti, Gianluigi Zanetti, Antonio Zorcolo \\ CRS4 Sardinia, Italy \\ Bruno Picasso \\ University of Cagliari, Italy
}

\begin{abstract}
The CRS4 experimental bone-burr simulator implements visual and haptic effects through the incorporation of a physics-based contact model and patient-specific data. Psychophysical tests demonstrate that, despite its simplified model and its inherent technological constraints, the simulator can articulate material differences, and that its users can learn to associate virtual bone with real bone material. Tests addressed both surface probing and interior drilling task. We also explore a haptic contrast sensitivity function based on the model's two main parameters: an elastic constant and an erosion factor. Both parameters manifest power-law-like sensitivity with respective exponents of around two and three. Further tests may reveal how well simulator users perceive fine differences in bone material, like those encountered while drilling through real volume boundaries.
\end{abstract}

Keywords: haptic, virtual reality, surgical simulator, psychophysics

\section{Introduction}

The temporal bone is one of our body's most complex anatomical areas [Nelson 1991]. Surgical access to its structures greatly extends the range of treatment for hearing and balance disorders. Yet, a high degree of dexterity, hands-on experience and anatomical knowledge are a training-prerequisite to effective temporal bone dissection. Since cadaver-based training is becoming increasingly problematic, teaching centers now seek to complement it with sessions on VR simulators-in particular, those designed to mimic patient-specific operating environments and provide realistic haptic and visual sensations. Early bone dissection simulators aimed to assist anatomical understanding by simply providing visualization of static models [Harada et al. 1988]. Recent simulators seek to include more realistic aspects for more practical tasks [Wiet et al. 2000; Bryan et al. 2001; Stredney et al. 2002; Pflesser et al. 2000; Pflesser et al. 2002]. Any realistic interactive bone-burr simulator, however, ought to simulate the physics of contact-detection and bone-erosion so as to generate appropriate haptic responses whilst also rendering secondary visual effects such as bone-debris accumulation, bleeding, irrigation and suction [Agus et al. 2002a]. The perceptual requirements on such a simulator impose stringent performance constraints in terms of both speed and accu-

*E-mail: gjb@crs4.it, Address: CRS4, Polaris, Loc.Piscinamanna Ed.1, CP-25,Pula (CA), I-09010 Italy. racy. Designed with such constraints in mind, the CRS4 experimental simulator [John et al. 2001; Agus et al. 2002b] implements semi-realistic visual and haptic effects through the incorporation of a physics-based contact model and patient-specific diagnostic data: two PHANToM devices govern the haptic interaction, while, to mimic real conditions, visualization is rendered on a binocular display-see figure 1.

This article focuses on evaluating how well the current simulator meets its perceptual requirements through the application of psychophysical tests. Ultimately, any such simulator should be judged on how well the experience it provides is transferred into practical surgical skills in real operations-as evaluated by the rate of successful outcomes. The tests that we report here can be considered as preliminary investigations carried out prior to such a full-scale evaluation. In them we aim to demonstrate that the prerequisite perceptual sensations are being adequately articulated by the simulator. Esen, Yano \& Buss [Esen et al. 2003] adopt a different approach by measuring the acquisition of controlled drilling skills by supervised trainees. Like them, however, we chose to measure participants insitu within the full visual-haptic virtual simulator. This is a practical necessity somewhat counter to the traditional practice of isolating the minimal stimuli needed to ascertain perceptual phenomena. The question of how perceptual information gets shared between our visual and haptic modalities [Newell et al. 2003] is not directly addressed here. We focus, instead, on measuring how well simulated material differences are distinguished during two basic tasks (a) surface probing - which elicits a tactile percept, and (b) drilling through a material - which elicits a haptic burring sensation. Expert surgeons report using these factors to perceive changes in bone material. Trainee surgeons traditionally practice on both real and artificial samples of temporal bone. The latter may vary from relatively crude PVC substitutes to sophisticated synthetic resin samples such as the Pettigrew Plastic Temporal Bones [Pettigrew ]. Here we investigate how well simulated rendering of these materials can be distinguished and to what extent they are preferentially associated with their real counterparts.

The CRS4 simulator embodies a parameterized physics-based model for bone material which is implemented via the available technology. Both the technological solution and physical model are fully described elsewhere [John et al. 2001; Agus et al. 2002b] - the following sections provide only a concise overview. Note, however, that each material is defined by its own particular parameter values. These values were initially set according to the subjective opinion expressed by three expert surgeon/users. They were later refined by objective experimental measurement using a custom-built dynamometric platform - incorporating load cells, strain gauges and a digital acquisition system - within which each real counterpart sample was vertically drilled, in turn, at constant applied force [Volpe and Khosla 1993; Schutter 1988]. 

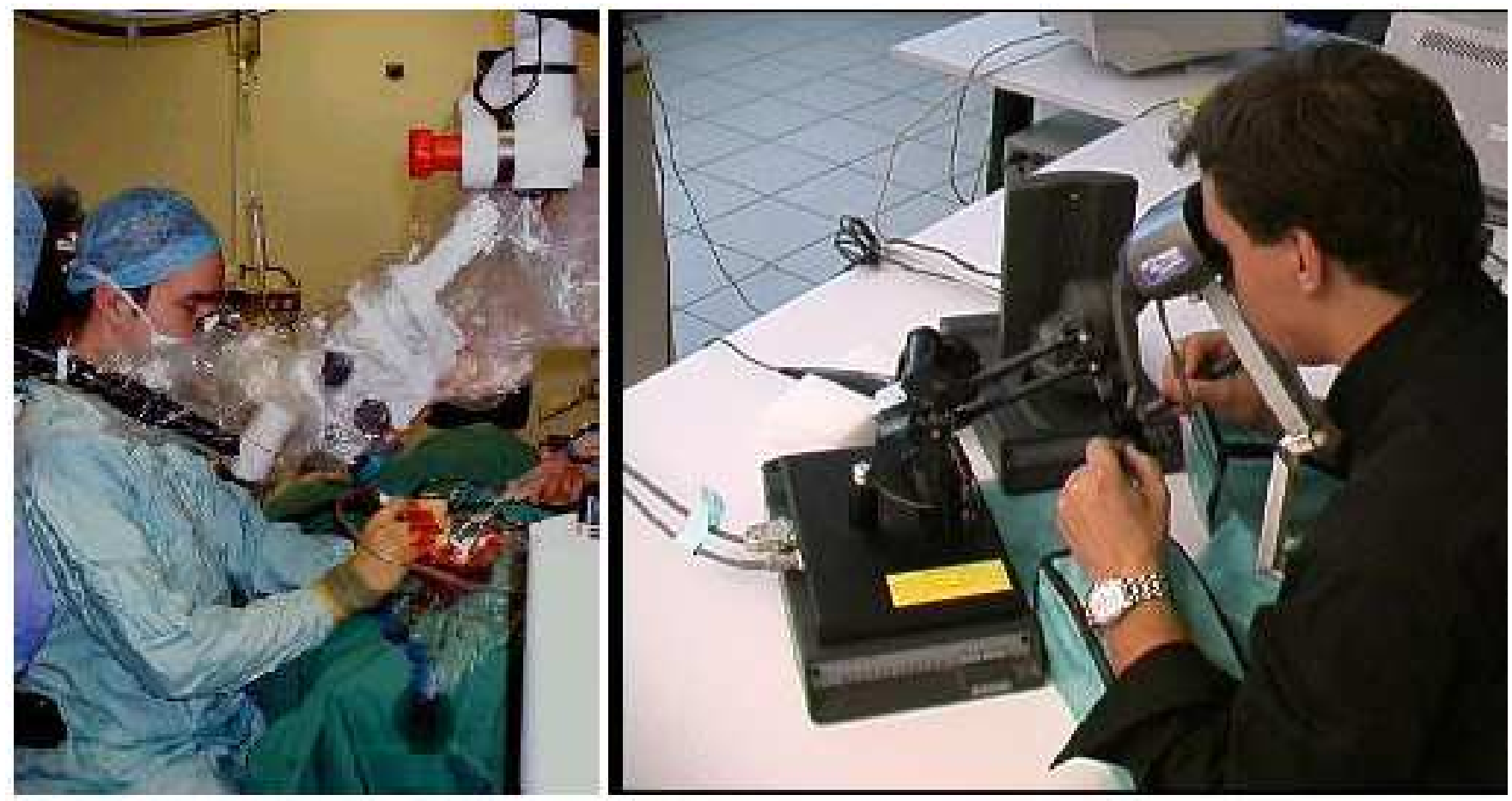

Figure 1: Surgical equipment and Simulator setup: Left: Real surgical environment, the surgeon keeps in his hands a high-speed rotating burr and a sucker and looks through a microscope. Right: The CRS4 simulator provides both haptic and binocular feedback.

\section{Technology overview}

The visual-haptic simulator, comprising both PHANToM devices and its binocular display is implemented, as a collection of loosely coupled concurrent components. The haptic component exploits a multi-resolution representation of the first two moments of the bone density to rapidly compute contact forces and determine bone erosion according to the physics-based model (see below). The visual component renders pre-acquired, patient-specific, anatomical data and uses a time-critical particle system evolution method to simulate the secondary visual effects - debris accumulation, bleeding, irrigation, and suction. The system runs on two interconnected multiprocessor machines. The data is initially replicated on the two machines. The first is dedicated to the high-frequency tasks: haptic device handling and bone removal simulation and run at 1 $\mathrm{KHz}$. The second runs concurrently at about $15-20 \mathrm{~Hz}$, the lowfrequency tasks: bone removal, fluid evolution and visual feedback. The two machines are synchronized via one-way message passing using the Virtual-Reality Peripheral Network (VRPN) library [Russell M. Taylor et al. 2001]. The VRPN system furnishes a deviceindependent and network-transparent interface to the virtual-reality peripherals. It is possible to record complete traces of the training sessions, like [Esen et al. 2003], for off-line by data analysis.

\section{Physics-based model}

The cutting of material by a rotating burr is complex since it involves: the tracking of the continuously changing free surface of the material being cut; the impact of the burr blades on the surface; the resulting stress distribution in the material; and the consequent plastic deformation and break-up. Therefore the standard methods found in the mechanical engineering literature for the simulation of milling cannot be applied. Thus we developed a simplified model, originally described in [Agus et al. 2002c], based on a limited number of parameters that were first tuned by trial and error by expert surgeons.

We assume that the burr-bit moves relatively slowly with respect to the time scale of the haptic feedback loop, and that the elastic forces exerted within the bone can be estimated by geometrically characterizing the region of bone intersected by an idealized sphere representing the burr tip.

Specifically, the burr-bit is modeled as a sphere of radius $R$ centered at $\mathbf{R}_{b}$, and we consider the first two moments, $m_{0}$ and $\mathbf{m}_{1}$, of the bone mass density, $\rho(\mathbf{r})$, contained within it.

$$
m_{0}=\int_{r<R} d r^{3} \rho(\mathbf{r}), \mathbf{m}_{1}=\int_{r<R} d r^{3} \rho(\mathbf{r}) \mathbf{r} .
$$

The direction of the local normal to the bone surface is then estimated as $\hat{\mathbf{n}}=-\mathbf{m}_{1} /\left|m_{1}\right|$. Then an effective "penetration depth" $h$ is derived from the quantity of mass, $m_{0}$, contained in the burr-bit as the smallest positive solution of

$$
m_{0}=\pi \rho_{0} R^{3}\left(\frac{h}{R}\right)^{2}\left(1-\frac{h}{3 R}\right)
$$

where $\rho_{0}$ is the "solid" bone reference density.

An effective force $\mathbf{F}_{e}$, intended to model the elastic response of the bone to the impinging burr can then be written:

$$
\mathbf{F}_{e}=c_{e} R^{2}(h / R)^{3 / 2} \hat{\mathbf{n}}
$$

where $c_{e}$ is a material constant, that describes the elastic properties. Note, this expression is consistent with Hertz's contact theory [Landau and Lifshitz 1986] in the limit when $h / R<<1$. 
The typical speed at which the burr-bit moves is less than $100 \mathrm{~mm} / \mathrm{s}$, while burr radii are generally between $1 \mathrm{~mm}$ and $5 \mathrm{~mm}$ [Agus et al. 2002a]. Since the haptic device acquisition period is $1 \mathrm{~ms}$, the burrbit will typically move just a small fraction of its radius. We thus compute interaction forces by checking collisions after the fact, rather than predicting them in advance.

Material removal in response to burring is modeled using a position dependent erosion rate described by $f$, an erosion shape function:

$$
\frac{d \rho(\mathbf{r})}{d t}=\alpha f(r / R) \rho(\mathbf{r})
$$

where, again, $\mathbf{r}$ is measured from the center of the burr-bit, and all necessary details of the burr-bit cutting characteristics, angular velocity, etc are combined in the single constant $\alpha$. Note, the shape function $f$ is constrained to have a maximum at $r / R=0$ and to be null where $r / R>1$. Note, the simulator also makes use of frictional and impact force models but these are not manipulated here.

The CRS4 simulator allows control of material properties by adjusting the elasticity constant in eq. 3 and the erosion constraint in eq. 4. Tabulated below are the values of $c_{e}$ and $\alpha$, fitted to our objective physical measurements of the three materials under investigation. Note, experts previously assigned $(1.5,0.1)$ to the Pettigrew plastic [Agus et al. 2002c; Agus et al. 2002a].

\begin{tabular}{|l|l|l|}
\hline$c_{e}$ & $\alpha$ & Material \\
\hline 2.145 & 0.087 & Real temporal bone \\
1.504 & 0.116 & Pettigrew plastic temporal bone [Pettigrew ] \\
0.462 & 0.256 & PVC K70 resin \\
\hline
\end{tabular}

Table 1: Elastic, $c_{e}$, and erosion, $\alpha$, model parameters.

\section{Psychophysics: Differentiating virtual materials}

Experts can sense the difference between real bone material and PVC by a combination of surface probing and interior drillingand the same ought also apply within the simulator. Here it was asked whether, using the same perceptual cues, our users are able to distinguish between virtual PVC and bone simulated using the parameter values from the appropriate rows in table 1 . Twenty volunteers with no previous experience with the simulator, or bonemilling, were recruited. After an initial phase of familiarization with the simulator, each was exposed to two sequences of 12 trials. In the first sequence the task was simply to probe the surface with the drill; in the second, it was to drill into the material's interior. Each trial was divided into two equal 20 s intervals following a two-alternative-forced-choice (2AFC) design. One of the two chosen material samples A or B was rendered in each interval. The subject was asked to indicate whether the material felt the same or different. The simulator was programmed to present samples in a random sequence while maintaining an equal number of trials presenting the four sample pairs: $\mathrm{AA}, \mathrm{BA}, \mathrm{AB}, \mathrm{BB}$-and the response of each subject to each test was recorded. The mean scores (out of 12) over all 20 subjects were:

1. Surface probing: $9.3+-0.4$

2. Drilling material: $9.6+-0.5$

Both these results exceed chance response level $(\mathrm{p}=0.001)$ and correspond to around a 75\% correct level-with some individuals obtaining perfect results and others worse as figure 2 shows. Clearly, with further training users would be expected to progressively improve their score, as in [Esen et al. 2003]. Using the same methodology described above, the 12 best performing subjects were then asked, by drilling, to differentiate between the simulated Pettigrew plastic and simulated temporal bone. Again, all subjects exceeded chance performance but, as expected, found it a little harder to differentiate the two materials-mean score (out of 12): $7.7+-1.4$.

\section{Psychophysics: Associating virtual and real bone}

A direct comparison was made between real bone milling and the simulated experience by requiring subjects to alternate between a real drilling task and the simulator. This was logistically more complex and the subject had to "keep in mind" the feel of the real material while subsequently comparing it to one of two simulated samples. Again a $2 \mathrm{AFC}$ design was employed: each trial comprised three intervals:

1. burring real Pettigrew plastic temporal bone,

2. using the simulator to burr virtual sample A for $10 \mathrm{~s}$,

3. using the simulator to burr virtual sample B for $10 \mathrm{~s}$,

where the length of the first interval was at subject's discretion. Since naive users generally found this task too confusing to participate usefully, we thus limited subjects to those already with a good grounding in simulated material difference: i.e. five that had performed well in the previous experiment. The task was to say which of the simulated samples A or B felt most like the real sample. In each trial the simulated samples were PVC and Pettigrew temporal bone material but presented in random order. In all, five subjects each performed 10 trials: three of whom scored 10 correct responses, while the remaining two got 8 and 9 right. Their mean score (out of 10) was $9.4+-0.4$, equivalent to $94 \%$ correct level, and clearly above chance $(\mathrm{p}=0.0001)$. This provides some preliminary indication that trained users perceive some correspondence between real and simulated bone materials.

\section{Psychophysics: Haptic sensitivity}

A more general approach may be to consider the simulator as a single system device and attempt to map out its haptic sensitivity in terms of the 2D parameter space $\left(c_{e}, \alpha\right)$ expressed by our model: i.e. elastic constant $v$. erosion factor. The operational region of this space lies within bounding limits defined-for the elastic constant between the sensation of "empty space" at $c_{e} \approx 0.1$ and that of "incapacitating vibration" felt at $c_{e} \approx 3.2$-and for the erosion factor as between "impenetrability" felt at $\alpha \approx 0.1$ and "zero resistance" at $\alpha \approx 1.0$. Although coordinates in this operating region do map to distinct stimuli they do not necessarily produce distinct percepts. For example, any closely neighboring pairs will be indistinguishable while widely separated points such as those marked as PVC and temporal bone in figure 3 ought to be easily differentiated. Between such extremes just noticeable differences (JNDs) will exist and may be mapped out by experiment. JNDs might map on to ellipses in $\left(c_{e}, \alpha\right)$ space, rather like they do in CIE chromaticity diagrams. In that cases they could feasibly be employed to construct a map of sensitivity across the space by accumulating measurements of perceived contrast between judiciously chosen stimulus pairs. Of course, this would require an exhaustive set of trials from a large set of subjects. Here we report an initial investigation to establish a few 


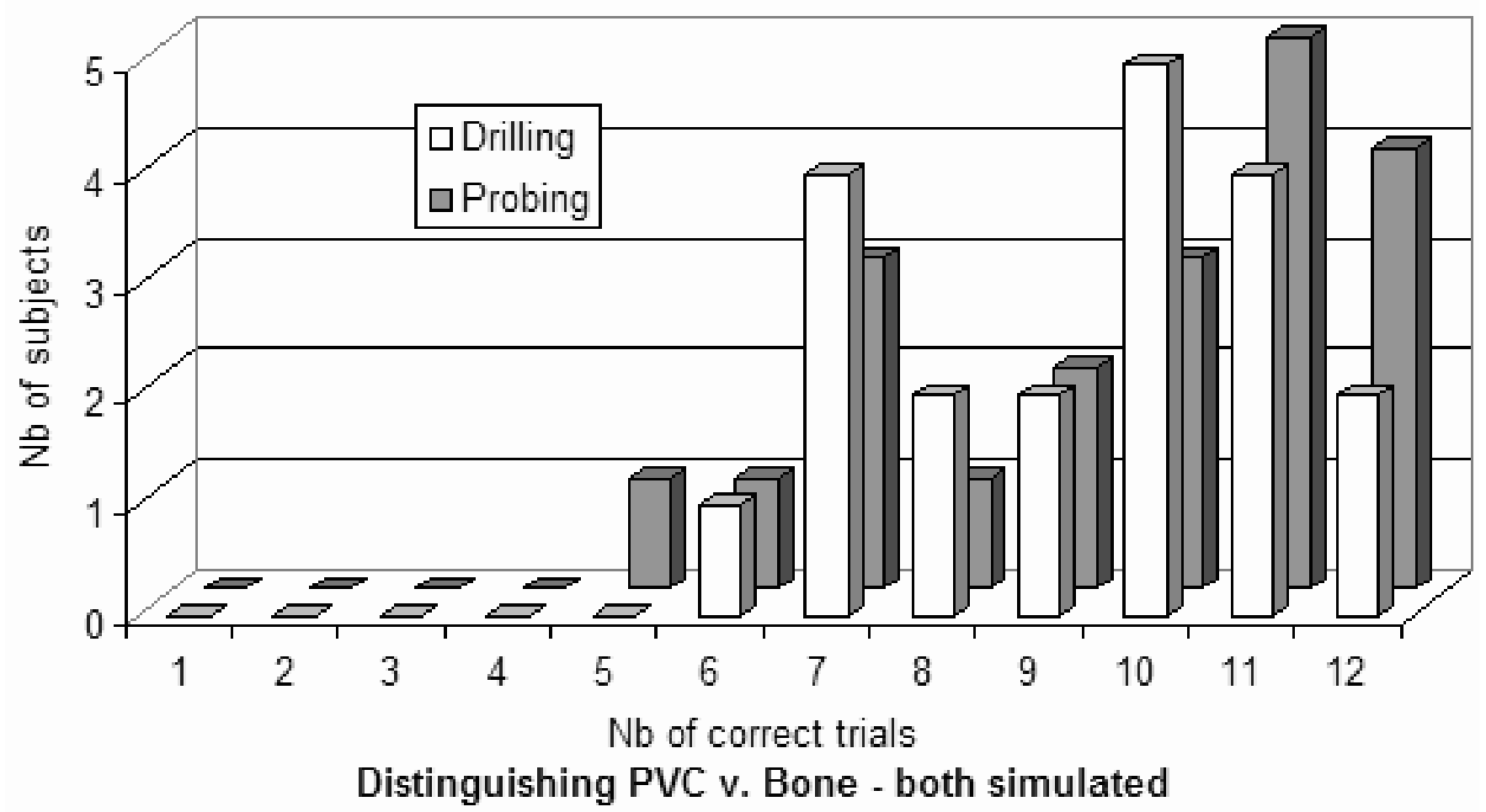

Figure 2: Histograms of results for both probing and drilling. Each shows the number of subjects that got a particular number of trials right. Note, no subject got less than five right.

representative JNDs in order to give some idea of how useful compiling such a contrast sensitivity map might be. Only if the JNDs are found to occupy loci that are fairly compact with respect to the bounded space will this approach be of much use. A method-ofadjustment protocol was applied to pairs of points scattered about the $\left(c_{e}, \alpha\right)$ space. Two experienced simulator users were employed as subjects. The distance between the points was adjusted until an approximate JND was established. This was done, initially, by fixing either $c_{e}$ or $\alpha$ and varying the other parameter. First results indicated that, regardless of the zone considered, it is fairly easy to distinguish a two-fold difference in the elastic constant, $c_{e}$; but a larger, three-fold difference is usually required in erosion factor, $\alpha$. Since the JNDs found seem to span multi-fold differences in the model parameter values, it may be more useful to plot contrast sensitivity using logarithmic axes, as we adopt in figure 3 .

\section{Discussion and Conclusion}

Plotting contrast sensitivity using logarithmic axes is equivalent to adopting a power-law basis as the haptic interface in our simulator-with exponents of approximately 2 and 3 for the parameters, $c_{e}$ and $\alpha$, respectively. We might have anticipated an exponential sensitivity relation for the elasticity factor by considering existing tactile Weber-Frechner or Steven's power law relations; however, this was less obvious for an erosion parameter. In any case, a more detailed mapping of contrast sensitivity might still produce some surprises since haptic feedback sensations stem from complex multiple receptive channels [Nicholson et al. 2000]: muscle contraction, joint movement, tactile and pressure receptors-the efferent output of which must be some how arbitrated and combined by the brain. Furthermore, these are not merely passive sensations since anticipatory motor cell activity always precedes the perception in highly correlated manner which may well introduce additional complexities.

Although, all the tests presented here are of a preliminary nature their results do generally indicate that the current CRS4 simulator is able to approximately articulate material difference through the setting of its model parameters. Users did learn to distinguish between three different materials and to preferentially associate virtual bone with real bone-despite the undoubtedly over-simplistic physicsbased model. Yet, it remains to be established how well they can perceive finer differences in bone material-especially those they may encounter, in drilling scenarios, at real volume boundaries. Given the relatively coarse contrast sensitivity characteristics (i.e. the broad JND loci) reported above, a further round of technological development might well be necessary before such fine differences can articulated. For an experimental demonstrator, such as the CRS4 simulator, intentionally built by integrating commercialoff-the-shelf components, there will always be an intrinsic technological compromise. It is particularly important for a medical simulator that any such compromise does not unduly prejudice the experience of the trainee. Psychophysical testing of the kind reported here remains an essential tool in the effective management of such compromise.

\section{Acknowledgment}

Thanks to Dr. Alastair Pettigrew for graciously providing Plastic Temporal bone samples; and to the NIH National Research Resource in Molecular Graphics and Microscopy at the University of N.Carolina at Chapel Hill for the VRPN library. 


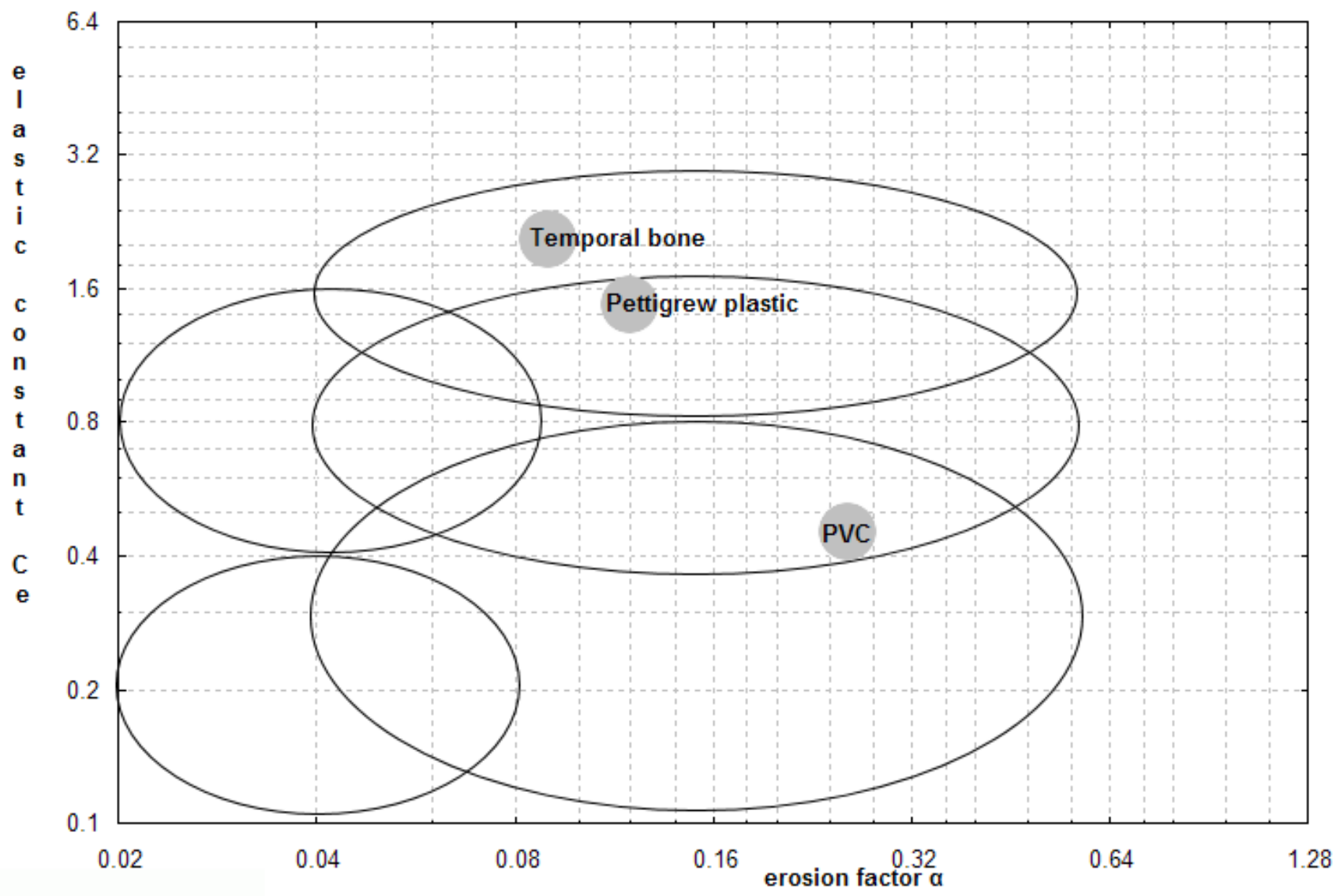

Figure 3: Contrast sensitivity space $\left(c_{e}, \alpha\right)$ plotted with logarithmic axes—showing grey dots: loci of three materials (bone, plastic and PVC); and ellipses: approximate JND loci measurements.

\section{References}

Agus, M., Giachetti, A., Gobbetti, E., Zanetti, G., John, N. W., AND StONE, R. J. 2002. Mastoidectomy simulation with combined visual and haptic feedback. In Medicine Meets Virtual Reality 2002, IOS Press, J. D. Westwood, H. M. Hoffmann, G. T. Mogel, and D. Stredney, Eds., 17-23.

Agus, M., Giachetti, A., Gobbetti, E., Zanetti, G., And ZorCOLO, A. 2002. A multiprocessor decoupled system for the simulation of temporal bone surgery. Computing and Visualization in Science 5, 1.

Agus, M., Giachetti, A., Gobbetti, E., Zanetti, G., And ZORCOLO, A. 2002. Real-time haptic and visual simulation of bone dissection. In IEEE Virtual Reality Conference, IEEE Computer Society Press, 209-216.

Bryan, J., Stredney, D., Wiet, G., and Sessanna, D. 2001. Virtual temporal bone dissection: A case study. In IEEE Visualization, 497-500.

EsEn, H., YANO, K., AND Buss, M. 2003. A control algorithm and preliminary user studies for a bone drilling medical training system. In RO-MAN Conference 2003.

Harada, T., IshiI, S., And TAYAma, N. 1988. Threedimensional reconstruction of the temporal bone from histological sections. Arch Otolaryngol Head Neck Surg 114, 1139-1142.
John, N. W., Thacker, N., Pokric, M., JaCKson, A., Zanetti, G., Gobbetti, E., Giachetti, A., Stone, R. J., Campos, J., Emmen, A., Schwerdtner, A., Neri, E., FrANCESChINI, S. S., AND RUBIO, F. 2001. An integrated simulator for surgery of the petrous bone. In Medicine Meets Virtual Reality 2001, IOS Press, J. D. Westwood, Ed., 218-224.

Landau, L., And Lifshitz, E. 1986. Theory of elasticity. Pergamon Press.

Nelson, R. A. 1991. Temporal Bone Surgical Dissection Manual, second ed. House Ear Institute, Los Angeles.

Newell, F., Bulthoff, H., AND ERnst, M. 2003. Cross-modal perception of actively explored objects. In Proc. EuroHaptics 2003, 291-299.

Nicholson, L., Adams, R., And Maher, C. 2000. Magnitude estimation of manually assessed elastic stiffness: Stability of the exponent. Perceptual and Motor Skills 91, 581-592.

Pettigrew, A. Pettigrew plastic temporal bones. http://www.temporal-bone.com/.

Pflesser, B., Petersik, A., Tiede, U., Hohne, K. H., AND LEUWER, R. 2000. Volume based planning and rehearsal of surgical interventions. In Computer Assisted Radiology and Surgery, Proc. CARS 2000, Excerpta Medica International Congress, H. U. L. et al., Ed., 1214, 607-612. 
Pflesser, B., Petersik, A., Tiede, U., Hohne, K. H., And LEUWER, R. 2002. Haptic volume interaction with anatomic models at sub-voxel resolution. In 10th International Symposium on Haptic Interfaces for Virtual Environment and Teleoperator Systems, Proc. Haptics 2002, 66-72.

Russell M. Taylor, l., Hudson, T. C., Seeger, A., WeBer, H., Juliano, J., AND Helser, A. T. 2001. Vrpn: a device-independent, network-transparent vr peripheral system. In Proceedings of the ACM symposium on Virtual reality software and technology, ACM Press, 55-61.

SCHUTTER, J. D. 1988. Improved force control laws for advanced tracking applications. In Proceedings of the IEEE Conference on Robotics and Automation, 1497-1502.

Stredney, D., Wiet, G., Bryan, J., Sessanna, D., MuRAKAMI, J., SCHAMLlbrock, O., POWEll, K., AND Welling, B. 2002. Temporal bone dissection simulation an update. In Medicine Meets Virtual Reality 2002, IOS Press, J. D. Westwood, H. M. Hoffmann, G. T. Mogel, and D. Stredney, Eds., 507-513.

Volpe, R., AND Khosla, P. 1993. Computational considerations in the implementation of force control strategies. Journal of Intelligent and Robotic Systems: Theory and Applications. Special Issue on Computational Aspects of Robot Kinematics, Dynamics, and Control..

Wiet, G., Bryan, J., Sessanna, D., Streadney, D., Schmalbrock, P., AND Welling, B. 2000. Virtual temporal bone dissection simulation. In Medicine Meets Virtual Reality 2000, IOS Press, J. D. Westwood, Ed., 378-384. 